\title{
$\beta$-Cyclodextrin-Propyl Sulfonic Acid Catalysed One-Pot Synthesis of 1,2,4,5-Tetrasubstituted Imidazoles as Local Anesthetic Agents
}

\author{
Yan Ran ${ }^{1, \dagger}$, Ming $\mathrm{Li}^{2, \dagger}$ and Zong-Ze Zhang ${ }^{1, *}$ \\ Received: 5 September 2015 ; Accepted: 7 October 2015 ; Published: 12 November 2015 \\ Academic Editors: Derek J. McPhee and Maria Emília de Sousa \\ 1 Zhongnan Hospital of Wuhan University, Department of Anesthesiology, Wuhan University, \\ Wuhan 430071, China; ranyanqwe@sina.com \\ 2 Department of Doppler Ultrasound, PLA421 Hospital, Guangzhou 510318, China; liming8782@sina.com \\ * Correspondence: zhongzongze@hotmail.com; Tel./Fax: +86-27-67812988 \\ $\dagger$ These authors contributed equally to this work.
}

\begin{abstract}
Some functionalized 1,2,4,5-tetrasubstituted imidazole derivatives were synthesized using a one-pot, four component reaction involving 1,2-diketones, aryl aldehydes, ammonium acetate and substituted aromatic amines. The synthesis has been efficiently carried out in a solvent free medium using $\beta$-cyclodextrin-propyl sulfonic acid as a catalyst to afford the target compounds in excellent yields. The local anesthetic effect of these derivatives was assessed in comparison to lidocaine as a standard using a rabbit corneal and mouse tail anesthesia model. The three most potent promising compounds were subjected to a rat sciatic nerve block assay where they showed considerable local anesthetic activity, along with minimal toxicity. Among the tested analogues, 4-(1-benzyl-4,5-diphenyl-1H-imidazol-2-yl)- $N, N$-dimethylaniline (5g) was identified as most potent analogue with minimal toxicity. It was further characterized by a more favourable therapeutic index than the standard.
\end{abstract}

Keywords: synthesis; imidazoles; local anesthetic agent

\section{Introduction}

Due to the ease of modification, diversity and effective synthetic options, heterocyclic compounds were considered as the preferred choice for the discovery of novel medications [1]. Of the many varieties available, compounds containing imidazole moieties have attracted considerable attention from medicinal chemists due to their wide array of medicinal properties [2], as it presents diverse pharmacological activities such as antimalarial [3], antibacterial [4], antihistaminic [5], anti-inflammatory [6], analgesic [7], antitubercular [8], antiprotozoal [9] and anthelmintic effects [10]. On the other hand, it has also found a special place in green chemistry as an ionic liquid component [11].

Therefore, extensive work has been carried on the development of efficient ways to prepare imidazoles. Particularly, four component syntheses involving condensation of a 1,2-diketone, a hydroxyketone or a ketomonoxime with an aldehyde, primary amine and ammonium acetate using $\mathrm{HY}$ zeolite [12], silica gel $/ \mathrm{NaHSO}_{4}$ [13], $\mathrm{HClO}_{4}-\mathrm{SiO}_{2}$ [14], molecular iodine [15], $\mathrm{BF}_{3}-\mathrm{SiO}_{2}$ [16], $\mathrm{InCl}_{3} \cdot 3 \mathrm{H}_{2} \mathrm{O}$ [17] or potassium dodecatugstocobaltatetrihydrate $\left(\mathrm{K}_{5} \mathrm{CoW}_{12} \mathrm{O}_{40} \cdot 3 \mathrm{H}_{2} \mathrm{O}\right)$ [18] as catalysts afforded 1,2,4,5-tetrasubstituted imidazoles in excellent yield. More recently, Ziarani et al., have reported the synthesis of 1,2,4,5-tetrasubstituted imidazoles in excellent yield under solvent free conditions using sulfonic acid functionalized silica $\left(\mathrm{SiO}_{2}-\mathrm{Pr}-\mathrm{SO}_{3} \mathrm{H}\right)$ as a catalyst [19]. However, the existing methods are not effective and are associated with several drawbacks, which include long 
reaction times, harsh reaction conditions, low yields of the products, tedious work-up procedures, and the use of toxic reagents.

Herein, we provide a green and economical one-pot synthesis of some 1,2,4,5-tetrasubstituted imidazoles catalysed by $\beta$-cyclodextrin-propyl sulfonic acid ( $\beta$-CD-PSA) as catalyst, as depicted in Scheme 1 . The products were also tested for local anesthetic activity using the rat tail flick and corneal hyperflexion methods.

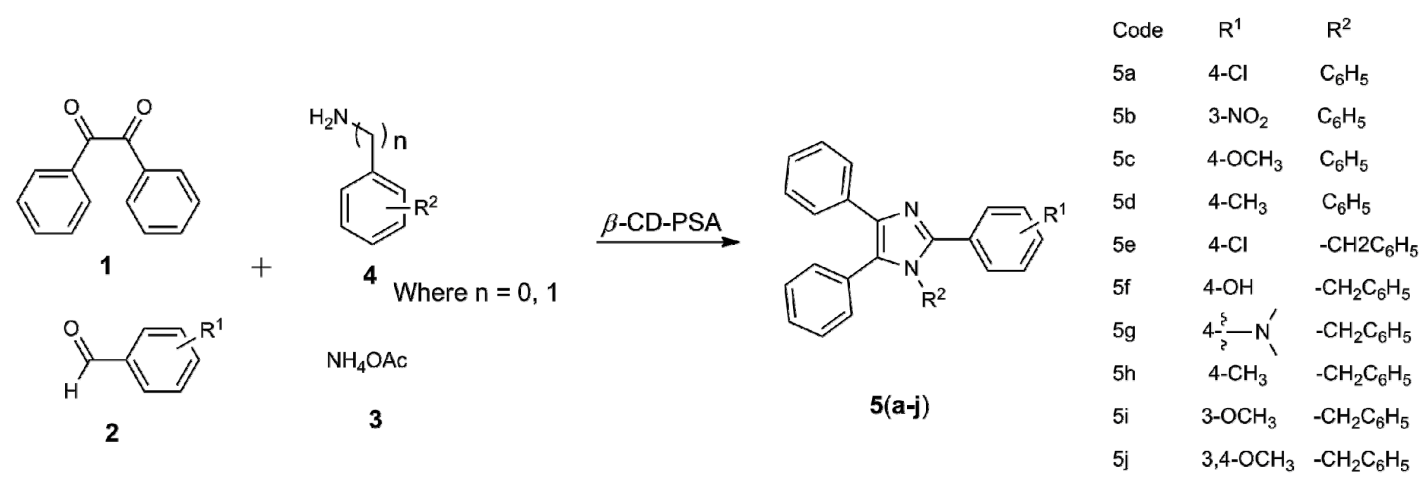

Scheme 1. One pot synthesis of 1,2,4,5-tetrasubstituted imidazoles catalysed by $\beta$-CD-PSA. Where $n=0$ means no spacer methylene fragment, i.e., aniline (5a-5d); $n=1$ means having the methylene spacer, i.e., benzylamine $(\mathbf{5 e}-\mathbf{5 j})$.

\section{Results and Discussion}

\subsection{Chemistry}

The synthesis of $\beta$-CD-PSA is shown in Scheme 2 . Initially, the commercially available $\beta$-CD was allowed to react with 1,3-propanesultone in $\mathrm{NaOH}$ solution to furnish sulfopropyl ether $\beta$-cyclodextrin (SPE- $\beta$-CD). This was further treated with an acidic resin to afford $\beta$-CD-PSA. The identification of $\beta$-CD-PSA was performed via spectroscopic methods and elemental analysis. The average degree of substitution in $\beta$-CD-PSA was estimated from ${ }^{1} \mathrm{H}-\mathrm{NMR}$ spectroscopy, and it was about 4.8. Moreover, the loading efficiency of propylsulfonic acid per g catalyst was also determined by elemental analysis as $2.45 \mathrm{mmol} / \mathrm{g}$.

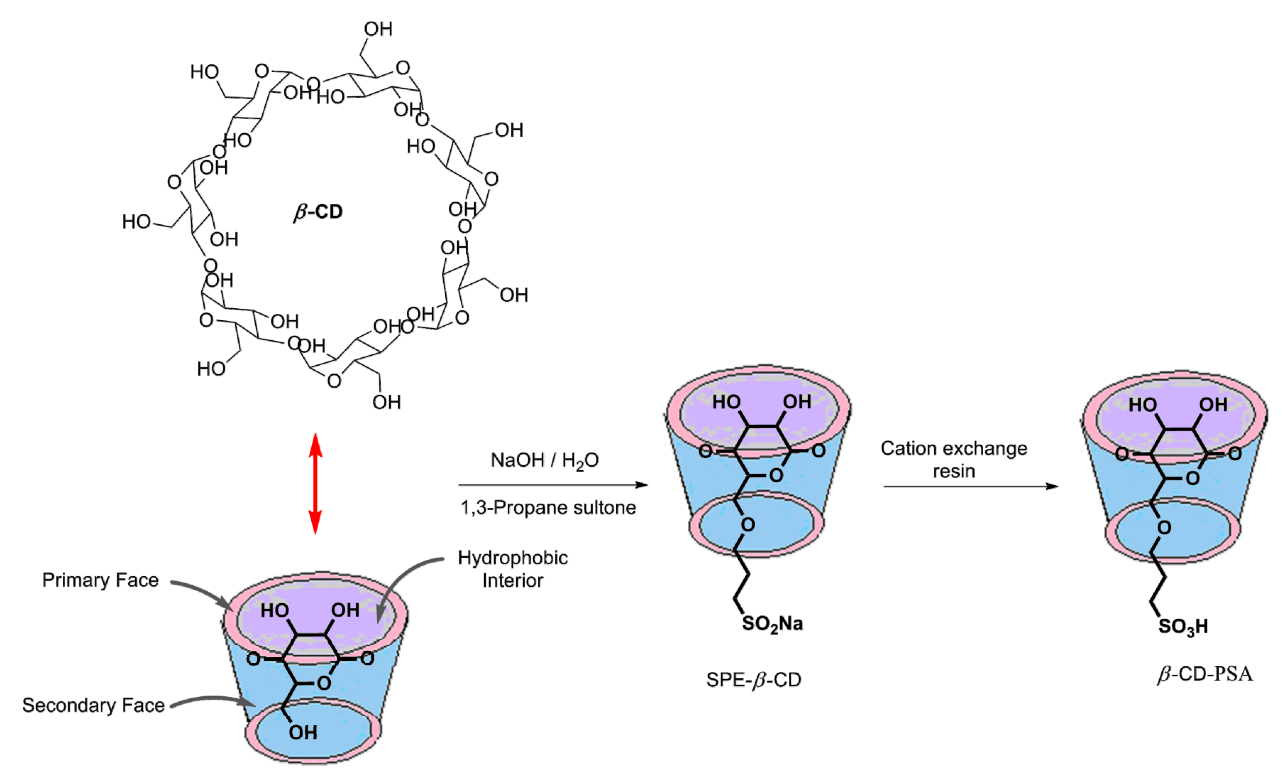

Scheme 2. The synthesis of $\beta$-CD-PSA. 
Next, to optimise the reaction conditions, initially the condensation reaction was examined using benzil (1 mmol), 4-chlorobenzaldehyde $(1 \mathrm{mmol})$, ammonium acetate $(3 \mathrm{mmol})$ and the catalyst $\beta$-CD-PSA to afford compound 5a (Scheme 1). As evident from Table 1, in the absence of catalyst at room temperature, the reaction does not proceed. Further, no change in reaction behaviour was noted on increasing the temperature. However, on introduction of the catalyst, the reaction proceeds well at room temperature to yield compound 5a. Significant improvement in product yield was reported on increasing the temperature up to $100{ }^{\circ} \mathrm{C}$, keeping the catalytic load constant, i.e., at $50{ }^{\circ} \mathrm{C}, 80^{\circ} \mathrm{C}$, and $100{ }^{\circ} \mathrm{C}$, the yield of product obtained is $55 \%, 85 \%$ and $96 \%$, respectively. This result suggests the role of catalyst and temperature for the completion of the reaction. In order to further confirm the role of $\beta$-CD-PSA, the next set of reactions (entries 7 and 8) was carried out at higher and lower catalyst concentration than $2 \mathrm{~mol} \%$. To our surprise, a significant decline in product yield was observed in both the cases, along with longer time being required for completion of the reaction. No further improvement in product yield was seen in water as well as in other solvents. It has thus been corroborated that the maximum yield of the product was obtained under solvent free conditions at $80{ }^{\circ} \mathrm{C}$ with a catalytic load of $2 \mathrm{~mol} \%$ of $\beta$-CD-PSA.

Table 1. Optimisation of reaction conditions for the synthesis of $5 \mathbf{a}^{\mathrm{a}}$.

\begin{tabular}{cccccc}
\hline Entry & Solvents & Temp $\left({ }^{\circ} \mathbf{C}\right)$ & Amount $(\mathbf{m o l}$ \%) & Time (min) & Yield (\%) b \\
\hline 1 & No Solvent & rt. & - & 150 & No reaction \\
2 & No Solvent & 80 & - & 150 & No reaction \\
3 & No Solvent & rt. & 2 & 150 & 28 \\
4 & No Solvent & 50 & 2 & 40 & 55 \\
5 & No Solvent & 80 & 2 & 30 & 85 \\
6 & No Solvent & 100 & 2 & 20 & 96 \\
7 & No Solvent & 80 & 1 & 70 & 74 \\
8 & No Solvent & 80 & 3 & 50 & 92 \\
9 & Water & 80 & 2 & 30 & 63 \\
10 & $\mathrm{CH}_{3} \mathrm{CH}{ }_{2} \mathrm{OH}$ & reflux & 2 & 40 & 78 \\
11 & $\mathrm{ClCH}_{2} \mathrm{CH} \mathrm{Cl}_{2}$ & reflux & 2 & 40 & 45 \\
12 & $\mathrm{EtOAc}$ & reflux & 2 & 40 & 70 \\
13 & $\mathrm{THF}$ & reflux & 2 & 40 & 56 \\
14 & $\mathrm{CH}_{3} \mathrm{CN}$ & 100 & 2 & 40 & 64 \\
15 & $\mathrm{DMF}$ & 100 & 2 & 40 & 72
\end{tabular}

a Reaction conditions: benzyl $(1 \mathrm{mmol})$, aromatic aldehyde $(1 \mathrm{mmol})$, ammonium acetate $(3 \mathrm{mmol})$, catalyst $\beta$-CD-PSA, solvent free or with solvent $(5 \mathrm{~mL}){ }^{\mathrm{b}}$ Isolated yield.

The next part of the study was aimed at comparing the efficiency of $\beta$-CD-PSA with other reported catalysts for the synthesis of 1,2,4,5-tetrasubstituted imidazoles. The results are shown in Table 2. It was found that the presence of catalyst together with high heat leads to the completion of the reactions to afford the target derivatives in considerable yield, accompanied by variations in the reaction time. Thus, it could be suggested that $\beta$-CD-PSA acts as a better catalyst in respect of reaction times and yields of the products than other previously used catalysts.

After successful optimisation of the reaction conditions, the next part of the work was focused on examining the versatility and limitations of this method using diverse substituents (Table 3). In the majority of the cases the reaction proceeds smoothly, for instance, compound 5a was furnished in the highest yield among all the derivatives. In the case of compound $5 \mathbf{b}$, the time required to complete the reaction was increased significantly, along with a minor reduction in yield. In general the reaction time was further increased in the case of compounds bearing electron withdrawing substituents i.e., entries 3 and 4. It is noteworthy to mention that, the yield of the product was decreased significantly along with extended reaction times with the introduction of a methylene bridge to connect phenyl at $\mathrm{R}_{2}$. Thus, it has been inferred that derivatives containing phenyl without the spacer were well suited to this condensation reaction to afford 1,2,4,5-tetrasubstituted imidazoles 
with varying reaction times and yields. On further comparing the results of these derivatives with the methods reported earlier ( $c f$. Table 2), it has been found that a variety of catalysts were used for the synthesis of the target compounds. The data presented in Table 2 suggests that, in the majority of cases, the reaction was carried out at higher temeperatures together with use of polar solvents such as ethanol, methanol, acetic acid, DMF and DMSO, leading to complex isolation and recovery procedures. Additionally, these processes generate waste containing catalyst and solvent, which have to be recovered, treated and disposed off. Moreover, the use of microwave irradiation prevents the synthesis of these compounds in resource-poor laboratories because of its expensive instrumentation. In this regard, the present methodology offers advantages over these other methods and furnisheds the desired compounds using greener aspects of efficient conditions without extensive purification. The structures of all newly compounds were ascertained on the basis of IR, ${ }^{1} \mathrm{H}-\mathrm{NMR},{ }^{13} \mathrm{C}-\mathrm{NMR}$ analysis, mass and elemental analysis and correlated with established M.P. reported in previous literatures as given in Table 3 (melting point).

Table 2. The comparison of efficiency of various catalysts for the synthesis of 1,2,4,5tetrasubstituted imidazoles ${ }^{\text {a }}$.

\begin{tabular}{|c|c|c|c|c|c|c|}
\hline Entry & Catalyst & Solvent & Condition & Yield (in \%) ${ }^{b}$ & Time & Ref. \\
\hline 1 & Zeolite HY & - & MW & $42-85$ & $6 \mathrm{~min}$ & [12] \\
\hline 2 & Silica gel & - & MW & $60-90$ & $6 \mathrm{~min}$ & [12] \\
\hline 3 & Silica gel/NaHSO 4 & DCM & reflux & $85-90$ & $2.5 \mathrm{~h}$ & [13] \\
\hline 4 & $\mathrm{HClO}_{4}-\mathrm{SiO}_{2}$ & - & $140^{\circ} \mathrm{C}$ & $60-94$ & $15-20 \mathrm{~min}$ & [14] \\
\hline 5 & $\mathrm{I}_{2}$ & Ethanol & $75^{\circ} \mathrm{C}$ & 97-99 & $15-20 \mathrm{~min}$ & [15] \\
\hline 6 & $\mathrm{~K}_{5} \mathrm{CoW}_{12} \mathrm{O}_{40} \cdot 3 \mathrm{H}_{2} \mathrm{O}$ & DCM & $140^{\circ} \mathrm{C}$ & $15-95$ & $2.5 \mathrm{~h}$ & {$[8]$} \\
\hline 7 & $\mathrm{BF}_{3} \cdot \mathrm{SiO}_{2}$ & - & $140^{\circ} \mathrm{C}$ & $80-96$ & $2 \mathrm{~h}$ & [16] \\
\hline 8 & $\mathrm{InCl}_{3} \cdot 3 \mathrm{H}_{2} \mathrm{O}$ & $\mathrm{MeOH}$ & r.t. & $47-84$ & $6-9 \mathrm{~h}$ & [17] \\
\hline 10 & {$\left[\left(\mathrm{CH}_{2}\right)_{4} \mathrm{SO}_{3} \mathrm{HMIM}\right]$} & - & $140^{\circ} \mathrm{C}$ & $85-95$ & $2-2.5 \mathrm{~h}$ & [20] \\
\hline 11 & MCM-41 & - & $140^{\circ} \mathrm{C}$ & $74-82$ & $1.92-2.25 \mathrm{~h}$ & [21] \\
\hline 12 & MCM-41 & $\mathrm{AcOH}$ & reflux & $75-85$ & $25-35 \min$ & [21] \\
\hline 13 & $p$-TsOH & - & $140^{\circ} \mathrm{C}$ & $75-82$ & $1.92-2.17 \mathrm{~h}$ & {$[21]$} \\
\hline 14 & $p$ - $\mathrm{TsOH}$ & $\mathrm{EtOH}$ & reflux & $73-83$ & $13-23 \mathrm{~min}$ & [21] \\
\hline 15 & - & 1-Butyl-3-methylimidazolium bromide & $140^{\circ} \mathrm{C}$ & $82-93$ & $1.5-5 \mathrm{~h}$ & [22] \\
\hline 16 & - & 1-Butyl-3-methylimidazolium bromide & MW & $82-93$ & $3-8 \min$ & [22] \\
\hline 17 & $\mathrm{P}_{2} \mathrm{O}_{5} / \mathrm{SiO}_{2}$ & - & $100^{\circ} \mathrm{C}$ & $87-98$ & $15-55 \mathrm{~min}$ & [23] \\
\hline 18 & - & - & $140^{\circ} \mathrm{C}$ & 0 & $3 \mathrm{~h}$ & {$[20]$} \\
\hline 19 & $\mathrm{SiO}_{2}-\mathrm{Pr}-\mathrm{SO}_{3} \mathrm{H}$ & - & $140^{\circ} \mathrm{C}$ & $85-98$ & $10 \mathrm{~min}-3 \mathrm{~h}$ & [19] \\
\hline 20 & $\beta-\mathrm{CD}-\mathrm{Pr}-\mathrm{SO}_{3} \mathrm{H}$ & - & $100^{\circ} \mathrm{C}$ & $68-96$ & $15 \mathrm{~min}-4 \mathrm{~h}$ & (This work) \\
\hline
\end{tabular}

Table 3. Synthesis of various 1,2,4,5-tetrasubstituted imidazoles catalysed by $\beta$-CD-PSA under solvent free conditions ${ }^{\text {a }}[20-24]$.

\begin{tabular}{|c|c|c|c|c|c|c|c|}
\hline Entry & $\mathbf{R}_{1}$ & $\mathbf{R}_{2}$ & Product & Time & Yield (in \%) ${ }^{b}$ & M.P. in ${ }^{\circ} \mathrm{C}$ (Observed) & M.P. in ${ }^{\circ} \mathrm{C}$ (in Literature) \\
\hline 1 & $4-\mathrm{Cl}$ & $\mathrm{C}_{6} \mathrm{H}_{5}$ & $5 a$ & $30 \mathrm{~min}$ & 96 & $158-160$ & $157-159$ \\
\hline 2 & $3-\mathrm{NO}_{2}$ & $\mathrm{C}_{6} \mathrm{H}_{5}$ & $5 b$ & $15 \mathrm{~min}$ & 93 & $247-249$ & $249-250$ \\
\hline 3 & $4-\mathrm{OCH}_{3}$ & $\mathrm{C}_{6} \mathrm{H}_{5}$ & $5 c$ & $3 \mathrm{~h}$ & 90 & 180-182 & $181-183$ \\
\hline 4 & $4-\mathrm{CH}_{3}$ & $\mathrm{C}_{6} \mathrm{H}_{5}$ & $5 d$ & $2.5 \mathrm{~h}$ & 90 & $188-190$ & $189-190$ \\
\hline 5 & $4-\mathrm{Cl}$ & $-\mathrm{CH}_{2} \mathrm{C}_{6} \mathrm{H}_{5}$ & $5 e$ & $3 \mathrm{~h}$ & 89 & $166-168$ & $163-165$ \\
\hline 6 & $4-\mathrm{OH}$ & $-\mathrm{CH}_{2} \mathrm{C}_{6} \mathrm{H}_{5}$ & $5 f$ & $3 \mathrm{~h}$ & 80 & $132-134$ & $131-132$ \\
\hline 7 & 4- $\{-N$ & $-\mathrm{CH}_{2} \mathrm{C}_{6} \mathrm{H}_{5}$ & $5 g$ & $3.5 \mathrm{~h}$ & 73 & 154-155 & $155-157$ \\
\hline 8 & $4-\mathrm{CH}_{3}$ & $-\mathrm{CH}_{2} \mathrm{C}_{6} \mathrm{H}_{5}$ & $5 \mathrm{~h}$ & $3.5 \mathrm{~h}$ & 75 & $160-162$ & $163-165$ \\
\hline 9 & $3-\mathrm{OCH}_{3}$ & $-\mathrm{CH}_{2} \mathrm{C}_{6} \mathrm{H}_{5}$ & $5 i$ & $4 \mathrm{~h}$ & 70 & $115-117$ & $128-129$ \\
\hline 10 & $3,4-\mathrm{OCH}_{3}$ & $-\mathrm{CH}_{2} \mathrm{C}_{6} \mathrm{H}_{5}$ & $5 \mathbf{j}$ & $4 \mathrm{~h}$ & 68 & 189-191 & 188-190 \\
\hline
\end{tabular}




\subsection{Reusability of the Catalyst}

Based on the above results and following the reaction optimization, we decided to explore the recyclability of $\beta$-CD-PSA taking the synthesis of $\mathbf{5 a}$ as a model reaction (Table 4 ). The catalyst was easily recovered upon filtration of the reaction mixture after addition of water. The filtrates were dried under vacuum and the resulting catalyst was reused directly for the next run. It has been found that the catalyst can be efficiently recycled for four consecutive runs with slight loss of activity. It was predicted that the catalyst recovery was efficient with a gradual decline in yield of product.

Table 4. Reusability of $\beta$-CD-PSA as catalyst for $\mathbf{5 a}$.

\begin{tabular}{cc}
\hline No. of Runs & Percentage Yield \\
\hline 1 & 96 \\
2 & 94 \\
3 & 90 \\
4 & 90 \\
\hline
\end{tabular}

\subsection{Pharmacology}

\section{Local Anesthetic Activity}

The target compounds were subjected to evaluation of local anesthetic activity on rabbit corneal and mouse tail anesthetic models which mimic surface and infiltration anesthesia. As shown in Table 5, except for compounds $\mathbf{5} \mathbf{g}$ and $\mathbf{5 i}$, none of the derivatives showed any significant activity in both the models. Compound $\mathbf{5 g}$ found to be more active than lidocaine used as a standard. In surface anesthesia, more than half the maximal response was observed in the case of compound $\mathbf{5 h}$. We can summarized that the presence of a benzylamine group has significant impact on the anesthetic potential of the compounds, while its replacement by aniline makes the compounds inactive.

Table 5. Local anesthetic evaluation of the target compounds in the rabbit corneal and mouse tail anesthetic models.

\begin{tabular}{ccc}
\hline Compound & Corneal Anesthesia $^{\mathbf{a}}$ & Mouse Tail Anesthesia $^{\mathbf{b}}$ \\
\hline $\mathbf{5 a}$ & $20.3 \pm 8.6$ & $11.3( \pm 0.11) \times 10^{-2}$ \\
$\mathbf{5 b}$ & $12.4 \pm 9.3$ & $5.7( \pm 0.42) \times 10^{-2}$ \\
$\mathbf{5} \mathbf{c}$ & $15.5 \pm 12.3$ & $6.5( \pm 0.23) \times 10^{-2}$ \\
$\mathbf{5 d}$ & $10.32 \pm 0.12$ & $4.3( \pm 0.54) \times 10^{-2}$ \\
$\mathbf{5 e}$ & $21.65 \pm 4.7$ & $3.4( \pm 0.32) \times 10^{-2}$ \\
$\mathbf{5 f}$ & $5.5 \pm 3.5$ & $5.4( \pm 0.14) \times 10^{-2}$ \\
$\mathbf{5 g}$ & $121.23 \pm 12.3$ & $1.6( \pm 0.26) \times 10^{-2}$ \\
$\mathbf{5 h}$ & $65.65 \pm 5.8$ & $9.2( \pm 0.16) \times 10^{-2}$ \\
$\mathbf{5 i}$ & $95.4 \pm 13.2$ & $7.4( \pm 0.18) \times 10^{-2}$ \\
$\mathbf{5 j}$ & $20.3 \pm 10.3$ & $4.1( \pm 0.45) \times 10^{-2}$ \\
Lidocaine HCl $^{\mathrm{c}}$ & 100 & $2.1( \pm 0.25) \times 10^{-2}$ \\
\hline
\end{tabular}

a All compounds were in aqueous solution at $2 \%$ concentration. The values, expressed as percentage of the anesthetic activity of lidocaine $(=100)$, are means \pm SE of three determinations. ${ }^{\mathrm{b}} \mathrm{IC}_{50}$ values expressed as $\mathrm{mol} / \mathrm{L} .{ }^{\mathrm{c}}$ Lidocaine hydrochloride was used as standard agent for comparison.

Owing to the excellent anesthetic activity, these compounds were further subjected to a rat sciatic nerve block assay for the determination of the duration of anesthetic activity. The result is shown Figure 1. It has been found that compound $5 \mathrm{~g}$ exhibits a similar anesthetic profile to that of the reference drug lidocaine. 


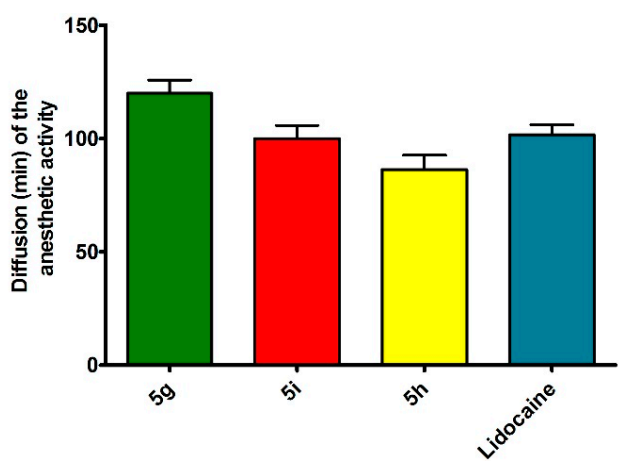

Figure 1. In vivo duration of local anesthetic activity (rat sciatic nerve block) of lidocaine and compounds $5 \mathrm{~g}, 5 \mathbf{i}$ and $5 \mathrm{~h}$. Each rat received $0.2 \mathrm{~mL}$ of the $2 \%$ anesthetic solution. The values are means \pm standard deviation of three independent experiments.

The compound $5 \mathrm{~g}$ was further subjected for the determination of the acute toxicity and therapeutic index in mice. As shown in Figure $2\left(\mathrm{LD}_{50}\right)$ and Figure $3\left(\mathrm{LD}_{50} / \mathrm{IC}_{50}\right)$, it is characterised by the most favourable ratio between toxicity and anesthetic activity. Moreover, the therapeutic index of the above compound was indicated as significantly higher than that of the lidocaine standard.

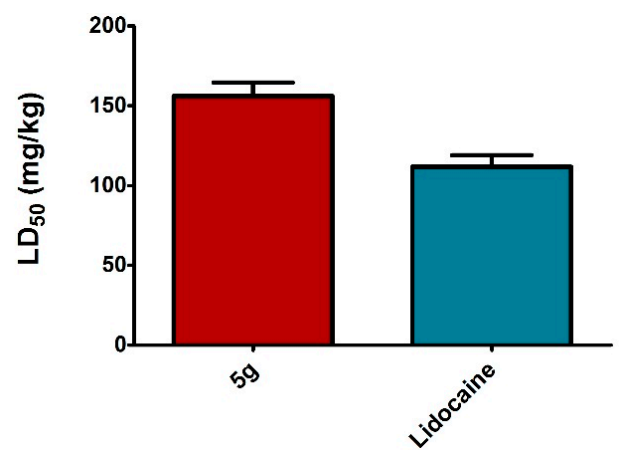

Figure 2. Acute toxicity of (ip) of lidocaine and compound $5 \mathbf{g}$ in male mice.

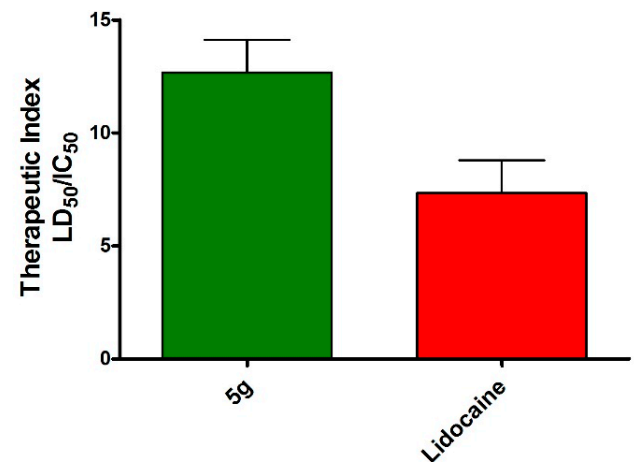

Figure 3. Therapeutic index of lidocaine and compound $5 \mathrm{~g}$ evaluated as ratio between $\mathrm{LD}_{50}$ and $\mathrm{IC}_{50}$ (mouse tail test expressed in $\mathrm{mg} / \mathrm{kg}$ ).

\section{Experimental Section}

\subsection{General Information}

All chemicals were of analytical grade and used directly. Melting points were determined in open capillary tubes with an Electrothermal melting point apparatus and are uncorrected. The completion of reaction was checked by thin layer chromatography (TLC) using silica gel-G coated 
Al-plates (0.5 mm thickness; Merck, Darmstadt, Germany) and the plates were illuminated under UV (254 nm) and evaluated in iodine vapour. FT-IR (in $2.0 \mathrm{~cm}^{-1}$, flat, smooth, abex, KBr) spectra were recorded on a Spectrum RX-I spectrometer (Perkin Elmer Instruments, Buckinghamshire, UK). Elemental analysis were determined on a Vario EL III CHNOS elemental analyzer (Elementar, Hanau, Germany). ${ }^{1} \mathrm{H}-\mathrm{NMR}$ spectra were recorded on a Bruker Avance II 400 NMR Spectrometer and the ${ }^{13} \mathrm{C}-\mathrm{NMR}$ spectra on a Bruker Avance II $100 \mathrm{NMR}$ spectrometer (Bruker Corporation, Billerica, MA, USA) in $\mathrm{D}_{2} \mathrm{O}$ or DMSO- $d_{6}$ using TMS as the internal standard. The chemical shifts are reported in parts per million (ppm, d), and signals are described as s (singlet), $d$ (doublet), $t$ (triplet), q (quartet), and $\mathrm{m}$ (multiplet). Mass spectra were obtained on a VG-AUTOSPEC spectrometer (VG Analytical, Manchester, UK).

\subsubsection{Synthesis of $\beta$-CD-PSA}

Initially, the $\beta-\mathrm{CD}(1 \mathrm{~g})$ was dissolved in $\mathrm{NaOH}$ solution $(5 \mathrm{M}, 10 \mathrm{~mL})$ at $75{ }^{\circ} \mathrm{C}$. To the above solution, 1,3-propane sultone (1.1 g) was added in small amounts and the mixture was stirred for $4 \mathrm{~h}$. The reaction solution was then cooled to room temperature and adjusted to neutral using $\mathrm{HCl}$ solution $(3 \mathrm{M})$. The mixture was poured in ethanol to afford sulfopropyl ether $\beta$-cyclodextrin $(\mathrm{SPE}-\beta-\mathrm{CD})$. In the next step, the acidic resin was activated in a saturated aqueous solution of $\mathrm{NaCl}$ for 1 day. This was followed by treatment with $2.5 \mathrm{wt} \% \mathrm{NaOH}$ aqueous solution for $80 \mathrm{~min}$, and then washing with distilled water until the $\mathrm{pH}$ reaches neutral. It was treated further with $5.0 \mathrm{wt} \% \mathrm{HCl}$ aqueous solution for $12 \mathrm{~h}$ and afterwards, the resin was transferred to a column and washed with deionized water until the eluent become neutral.

Finally, the sodium salt of SPE- $\beta-C D(0.5 \mathrm{~g})$ was dissolved in water $(100 \mathrm{~mL})$, and the solution was allowed to flow through the acidic resin column at a speed of 20 drops per minute. The acidic eluent was collected and then freeze-dried for $12 \mathrm{~h}$ to obtain the $\beta$-CD-PSA product. FT-IR $\left(v_{\max }\right.$; $\left.\mathrm{cm}^{-1} \mathrm{KBr}\right): 3354,2930,2365,1653,1361,1153,1030,700 .{ }^{1} \mathrm{H}-\mathrm{NMR}\left(400 \mathrm{MHz}, \mathrm{CDCl}_{3}\right) \delta, \mathrm{ppm}:$ 5.01-5.11 (m, C1-H), 3.49-3.81 (m, $\left.-\mathrm{OCH}_{2} \mathrm{CH}_{2} \mathrm{CH}_{2} \mathrm{SO}_{3} \mathrm{H}, \mathrm{CH}\right), 2.85\left(\mathrm{~s},-\mathrm{OCH}_{2} \mathrm{CH}_{2} \mathrm{CH}_{2} \mathrm{SO}_{3} \mathrm{H}\right), 2.28$ (s, $\left.-\mathrm{OCH}_{2} \mathrm{CH}_{2} \mathrm{CH}_{2} \mathrm{SO}_{3} \mathrm{H}\right) ;{ }^{13} \mathrm{C}-\mathrm{NMR}\left(100 \mathrm{MHz}, \mathrm{D}_{2} \mathrm{O}\right): \delta$, ppm: 80.9, 72.7, 71.6, 69.4, 67.8, 53.2, 28.2, 27.8, 21.1, 19.9; Anal. Found: C 33.52, S 7.834, H 6.67.

\subsubsection{General Procedure for the Synthesis of 1,2,4,5-Tetrasubstituted Imidazoles 5(a-j)}

The catalyst $\beta$-CD-PSA $(0.02 \mathrm{~mol})$, an aromatic aldehyde $(1 \mathrm{mmol})$, benzil $(1 \mathrm{mmol})$, aniline or benzylamine $(1 \mathrm{mmol})$ and ammonium acetate $(3 \mathrm{mmol})$ were placed in a flask and stirred at $100{ }^{\circ} \mathrm{C}$ under solvent free conditions for a suitable time (Table 3). The progress of the reaction was monitored by TLC. After completion of the reaction, ethyl acetate $(5 \mathrm{~mL})$ was added to the reaction mixture, which was filtered to remove the insoluble catalyst. The crystals of 1,2,4,5-tetrasubstituted imidazoles appeared after gradual evaporation of solvent at room temperature. The solution of $\beta$-CD-PSA was dried under vacuum for recycling the catalyst in the next run, and can be used several times without much loss of catalytic activity.

2-(4-Chlorophenyl)-1,4,5-triphenyl-1H-imidazole (5a). MW: 406.91; $\mathrm{R}_{f}: 0.75 ;$ FT-IR $\left(v_{\max } \mathrm{cm}^{-1}\right.$ $\mathrm{KBr}): 3038,2979\left(\mathrm{C}-\mathrm{H}_{\text {stretch }}\right), 1648\left(\mathrm{C}=\mathrm{N}_{\text {stretch }}\right), 1215\left(\mathrm{C}-\mathrm{N}_{\text {stretch }}\right), 891$ (aromatic-Cl) cm ${ }^{-1} ;{ }^{1} \mathrm{H}-\mathrm{NMR}$ $\left(400 \mathrm{MHz}, \mathrm{CDCl}_{3}\right) \delta$, ppm: 8.09-8.04 (d, $\left.2 \mathrm{H} \mathrm{J}=8.4 \mathrm{~Hz}, \mathrm{Ar}-\mathrm{H}\right), 7.79-7.76(\mathrm{~d}, 2 \mathrm{H} \mathrm{J}=8.1 \mathrm{~Hz}, \mathrm{Ar}-\mathrm{H})$, 7.58-7.41 (m, 15H, Ar-H); ${ }^{13} \mathrm{C}-\mathrm{NMR}\left(100 \mathrm{MHz}, \mathrm{CDCl}_{3}\right)$ \&, ppm: 144.2, 141.3, 138.8, 138.2, 134.3, 133.1, 131.8, 129.7, 129.3, 129.2, 128.9, 128.7, 128.2, 127.5, 122.1; Mass spectrum: $407.89(\mathrm{M}+1)$; Elemental analysis for $\mathrm{C}_{27} \mathrm{H}_{19} \mathrm{ClN}_{2}$ : Calculated: $\mathrm{C}, 79.70 ; \mathrm{H}, 4.71 ; \mathrm{N}, 6.88$; Found: $\mathrm{C}, 79.68 ; \mathrm{H}, 4.71 ; \mathrm{N}, 6.89$.

2-(3-Nitrophenyl)-1,4,5-triphenyl-1H-imidazole (5b). MW: 417.46; $\mathrm{R}_{f}$ : 0.63; FT-IR ( $\left.v_{\max } ; \mathrm{cm}^{-1} \mathrm{KBr}\right)$ : 3045, $2972\left(\mathrm{C}-\mathrm{H}_{\text {stretch }}\right), 1651\left(\mathrm{C}=\mathrm{N}_{\text {stretch }}\right), 1213\left(\mathrm{C}-\mathrm{N}_{\text {stretch }}\right), 878$ (aromatic- $\left.-\mathrm{NO}_{2}\right) \mathrm{cm}^{-1} ;{ }^{1} \mathrm{H}-\mathrm{NMR}(400 \mathrm{MHz}$, $\mathrm{CDCl}_{3}$ ) $\delta$, ppm: 8.58-8.53 (d, 2H J = 8.2 Hz, Ar-H), 7.94-7.91 (d, 2H J = 7.6 Hz, Ar-H), 7.73-7.42 (m, 15H, Ar-H); ${ }^{13} \mathrm{C}-\mathrm{NMR}\left(100 \mathrm{MHz}, \mathrm{CDCl}_{3}\right) \delta$, ppm: 148.5, 144.4, 138.7, 138.2, 133.7, 133.1, 131.7, 131.4, 
130.2, 129.6, 129.2, 128.7, 128.2, 127.5, 123.9, 122.8, 122.1; Mass spectrum: $418.48(\mathrm{M}+1)$; Elemental analysis for $\mathrm{C}_{27} \mathrm{H}_{19} \mathrm{~N}_{3} \mathrm{O}_{2}$ : Calculated: $\mathrm{C}, 77.68 ; \mathrm{H}, 4.59 ; \mathrm{N}, 10.07$; Found: $\mathrm{C}, 77.69 ; \mathrm{H}, 4.61 ; \mathrm{N}, 10.08$.

2-(4-Methoxyphenyl)-1,4,5-triphenyl-1H-imidazole (5c). MW: 402.49; $\mathrm{R}_{f}$ : 0.78; FT-IR $\left(v_{\max } \mathrm{cm}^{-1} \mathrm{KBr}\right)$ : 3052, $2971\left(\mathrm{C}-\mathrm{H}_{\text {stretch }}\right), 1653\left(\mathrm{C}=\mathrm{N}_{\text {stretch }}\right), 1218\left(\mathrm{C}-\mathrm{N}_{\text {stretch }}\right), 892 \mathrm{~cm}^{-1} ;{ }^{1} \mathrm{H}-\mathrm{NMR}\left(400 \mathrm{MHz}, \mathrm{CDCl}_{3}\right) \delta$, ppm: 7.98-7.94 (d, 2H J = 8.3 Hz, Ar-H), 7.79-7.41 (m, 15H, Ar-H), 7.04-7.01 (d, 2H J = 6.3 Hz, Ar-H), 3.87 (s, 3H, $\left.\mathrm{OCH}_{3}\right) ;{ }^{13} \mathrm{C}-\mathrm{NMR}\left(100 \mathrm{MHz}, \mathrm{CDCl}_{3}\right) \delta$, ppm: 160.6, 144.4, 138.7, 138.2, 135.5, 133.1, 131.7, 130.4, 129.7, 129.2, 128.7, 128.1, 127.5, 122.2, 114.8, 55.9; Mass spectrum: $403.49(\mathrm{M}+1)$; Elemental analysis for $\mathrm{C}_{28} \mathrm{H}_{22} \mathrm{~N}_{2} \mathrm{O}$ : Calculated: $\mathrm{C}, 83.56 ; \mathrm{H}, 5.51 ; \mathrm{N}, 6.96$; Found: $\mathrm{C}, 83.58 ; \mathrm{H}, 5.52 ; \mathrm{N}, 6.97$.

1,4,5-Triphenyl-2-(p-tolyl)-1H-imidazole (5d). MW: 386.49; $\mathrm{R}_{f}$ : 0.65; FT-IR ( $\left.v_{\max } ; \mathrm{cm}^{-1} \mathrm{KBr}\right)$ : 3059, $2968\left(\mathrm{C}-\mathrm{H}_{\text {stretch }}\right), 1657\left(\mathrm{C}=\mathrm{N}_{\text {stretch }}\right), 1214\left(\mathrm{C}-\mathrm{N}_{\text {stretch }}\right), 899 \mathrm{~cm}^{-1},{ }^{1} \mathrm{H}-\mathrm{NMR}\left(400 \mathrm{MHz}, \mathrm{CDCl}_{3}\right) \delta, \mathrm{ppm}$ : 8.58-8.56 (d, 2H J = 8.4 Hz, Ar-H), 7.78-7.43 (m, 15H, Ar-H), 7.28-7.24 (d, 2H J = 6.7 Hz, Ar-H), 2.35 (s, 3H, $\left.\mathrm{CH}_{3}\right) ;{ }^{13} \mathrm{C}-\mathrm{NMR}\left(100 \mathrm{MHz}, \mathrm{CDCl}_{3}\right) \delta$, ppm: 144.5, 140.2, 138.7, 138.2, 133.1, 131.7, 129.7, 129.4, 129.1, 128.7, 128.1, 127.5, 125.7, 122.7, 21.3; Mass spectrum: $387.51(\mathrm{M}+1)$; Elemental analysis for $\mathrm{C}_{28} \mathrm{H}_{22} \mathrm{~N}_{2}$ : Calculated: $\mathrm{C}, 87.01 ; \mathrm{H}, 5.74 ; \mathrm{N}, 7.25$; Found: $\mathrm{C}, 87.03 ; \mathrm{H}, 5.74 ; \mathrm{N}, 7.26$.

1-Benzyl-2-(4-chlorophenyl)-4,5-diphenyl-1H-imidazole (5e). MW: 420.93; $\mathrm{R}_{f}$ : 0.64; FT-IR $\left(v_{\max } \mathrm{cm}^{-1}\right.$ $\mathrm{KBr}): 3063,2969\left(\mathrm{C}-\mathrm{H}_{\text {stretch }}\right), 1654\left(\mathrm{C}=\mathrm{N}_{\text {stretch }}\right), 1211\left(\mathrm{C}-\mathrm{N}_{\text {stretch }}\right), 893(\mathrm{Cl}) \mathrm{cm}^{-1}{ }^{-1} \mathrm{H}-\mathrm{NMR}(400 \mathrm{MHz}$, $\mathrm{CDCl}_{3}$ ) $\delta$, ppm: 8.12-8.09 (d, 2H J = 8.2 Hz, Ar-H), 7.78-7.23 (m, 15H, Ar-H), 7.48-7.44 (d, 2H $J=6.2 \mathrm{~Hz}, \mathrm{Ar}-\mathrm{H}), 5.59$ (s, 2H, $\left.\mathrm{CH}_{2}\right) ;{ }^{13} \mathrm{C}-\mathrm{NMR}\left(100 \mathrm{MHz}, \mathrm{CDCl}_{3}\right) \delta$, ppm: 153.7, 141.4, 138.2, 137.3, 134.3, 133.1, 129.6, 129.4, 129.1, 128.9, 128.4, 128.2, 127.6, 127.5, 125.7, 47.5; Mass: $421.94(\mathrm{M}+1)$; Elemental analysis for $\mathrm{C}_{28} \mathrm{H}_{21} \mathrm{ClN}_{2}$ : Calculated: $\mathrm{C}, 79.89 ; \mathrm{H}, 5.03 ; \mathrm{N}, 6.66$; Found: $\mathrm{C}, 79.91 ; \mathrm{H}, 5.03$; $\mathrm{N}, 6.66$.

4-(1-Benzyl-4,5-diphenyl-1H-imidazol-2-yl)phenol (5f). MW: 402.49; $\mathrm{R}_{f}$ : 0.51; FT-IR $\left(v_{\max } ; \mathrm{cm}^{-1} \mathrm{KBr}\right)$ : 3068, $2962\left(\mathrm{C}-\mathrm{H}_{\text {stretch }}\right), 1657\left(\mathrm{C}=\mathrm{N}_{\text {stretch }}\right), 1218\left(\mathrm{C}-\mathrm{N}_{\text {stretch }}\right), 897 \mathrm{~cm}^{-1} ;{ }^{1} \mathrm{H}-\mathrm{NMR}\left(400 \mathrm{MHz}, \mathrm{CDCl}_{3}\right) \delta$, ppm: 7.91-7.89 (d, 2H J = 8.4 Hz, Ar-H), 7.79-7.23 (m, 15H, Ar-H), 6.89-6.84 (d, 2H J = 6.7 Hz, Ar-H), $5.58\left(\mathrm{~s}, 2 \mathrm{H}, \mathrm{CH}_{2}\right), 5.28(\mathrm{~s}, 1 \mathrm{H}, \mathrm{OH}) ;{ }^{13} \mathrm{C}-\mathrm{NMR}\left(100 \mathrm{MHz}, \mathrm{CDCl}_{3}\right) \delta$, ppm: 158.5, 153.7, 141.3, 138.2, 137.3, 133.1, 130.7, 129.6, 129.1, 128.8, 128.4, 127.6, 127.3, 125.7, 123.2, 116.4, 47.5; Mass spectrum: $403.51(\mathrm{M}+1)$; Elemental analysis for $\mathrm{C}_{28} \mathrm{H}_{22} \mathrm{~N}_{2} \mathrm{O}$ : Calculated: $\mathrm{C}, 83.56 ; \mathrm{H}, 5.51 ; \mathrm{N}, 6.96$; Found: C, 83.58; H, 5.52; N, 6.96 .

4-(1-Benzyl-4,5-diphenyl-1H-imidazol-2-yl)-N,N-dimethylaniline (5g). MW: 429.56; $\mathrm{R}_{f}$ : 0.61; FT-IR ( $v_{\max }$;

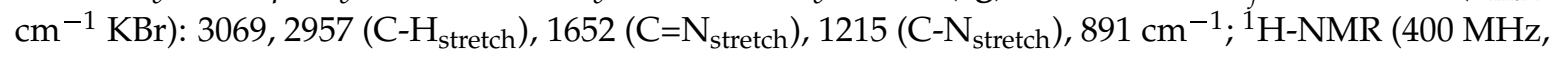
$\left.\mathrm{CDCl}_{3}\right) \delta$, ppm: 7.97-7.95 (d, 2H J = 8.2 Hz, Ar-H), 7.79-7.24 (m, 15H, Ar-H), 6.82-6.79 (d, 2H $J=6.4 \mathrm{~Hz}, \mathrm{Ar}-\mathrm{H}), 5.58\left(\mathrm{~s}, 2 \mathrm{H}, \mathrm{CH}_{2}\right), 3.06\left(\mathrm{~s}, 6 \mathrm{H}, \mathrm{CH}_{3} \times 2\right) ;{ }^{13} \mathrm{C}-\mathrm{NMR}\left(100 \mathrm{MHz}, \mathrm{CDCl}_{3}\right) \delta$, ppm: 155.3, 153.7, 141.3, 138.2, 137.3, 133.1, 129.6, 129.2, 128.7, 128.4, 128.1, 127.6, 127.2, 125.7, 120.2, 112.8, 47.5, 41.3; Mass spectrum: $430.58(\mathrm{M}+1)$; Elemental analysis for $\mathrm{C}_{30} \mathrm{H}_{27} \mathrm{~N}_{3}$ : Calculated: $\mathrm{C}, 83.88$; H, 6.34; N, 9.78; Found: C, 83.91; H, 6.33; N, 9.78.

1-Benzyl-4,5-diphenyl-2-(p-tolyl)-1H-imidazole (5h). MW: 400.51; $\mathrm{R}_{f}$ : 0.53; FT-IR ( $\left.v_{\max } ; \mathrm{cm}^{-1} \mathrm{KBr}\right)$ : 3069, $2956\left(\mathrm{C}-\mathrm{H}_{\text {stretch }}\right), 1659\left(\mathrm{C}=\mathrm{N}_{\text {stretch }}\right), 1218\left(\mathrm{C}-\mathrm{N}_{\text {stretch }}\right), 887 \mathrm{~cm}^{-1} ;{ }^{1} \mathrm{H}-\mathrm{NMR}\left(400 \mathrm{MHz}, \mathrm{CDCl}_{3}\right) \delta, \mathrm{ppm}$ : 8.48-8.46 (d, $2 \mathrm{H} \mathrm{J}=8.4 \mathrm{~Hz}, \mathrm{Ar}-\mathrm{H}), 7.79-7.22(\mathrm{~m}, 15 \mathrm{H}, \mathrm{Ar}-\mathrm{H}), 7.28-7.24(\mathrm{~d}, 2 \mathrm{H} \mathrm{J}=6.1 \mathrm{~Hz}, \mathrm{Ar}-\mathrm{H}), 5.58$ (s, 2H, CH 2$), 2.28$ (s, 3H, $\left.\mathrm{CH}_{3}\right) ;{ }^{13} \mathrm{C}-\mathrm{NMR}\left(100 \mathrm{MHz}, \mathrm{CDCl}_{3}\right)$, ppm: 153.7, 141.3, 138.2, 137.3, 133.1, 131.7, 129.8, 129.5, 129.1, 128.7, 128.3, 127.6, 127.2, 125.7, 47.5, 21.3; Mass spectrum: $401.48(\mathrm{M}+1)$; Elemental analysis for $\mathrm{C}_{29} \mathrm{H}_{24} \mathrm{~N}_{2}$ : Calculated: $\mathrm{C}$, 86.97; H, 6.04; N, 6.99; Found: C, 86.98; H, 6.04; N, 6.98 .

1-Benzyl-2-(4-methoxyphenyl)-4,5-diphenyl-1H-imidazole (5i). MW: 416.51; $\mathrm{R}_{f}: 0.65 ;$ FT-IR $\left(v_{\max } ; \mathrm{cm}^{-1}\right.$ $\mathrm{KBr}): 3069,2959\left(\mathrm{C}-\mathrm{H}_{\text {stretch }}\right), 1655\left(\mathrm{C}=\mathrm{N}_{\text {stretch }}\right), 1216\left(\mathrm{C}-\mathrm{N}_{\text {stretch }}\right), 884 \mathrm{~cm}^{-1} ;{ }^{1} \mathrm{H}-\mathrm{NMR}(400 \mathrm{MHz}$, $\left.\mathrm{CDCl}_{3}, \mathrm{TMS}\right) \delta$, ppm: 7.97-7.96 (d, 2H J = 8.3 Hz, Ar-H), 7.79-7.23 (m, 15H, Ar-H), 7.05-7.02 (d, $2 \mathrm{H} \mathrm{J}=6.4 \mathrm{~Hz}, \mathrm{Ar}-\mathrm{H}), 5.58\left(\mathrm{~s}, 2 \mathrm{H}, \mathrm{CH}_{2}\right), 3.82\left(\mathrm{~s}, 3 \mathrm{H}, \mathrm{OCH}_{3}\right) ;{ }^{13} \mathrm{C}-\mathrm{NMR}\left(100 \mathrm{MHz}, \mathrm{CDCl}_{3}\right) \delta$, ppm: $160.6,153.7,141.2,138.3,137.3,133.1,130.2,129.7,129.2,128.8,128.3,127.8,127.4,125.6,122.8,114.8$, 
55.7, 47.5; Mass spectrum: $417.51(\mathrm{M}+1)$; Elemental analysis for $\mathrm{C}_{29} \mathrm{H}_{24} \mathrm{~N}_{2} \mathrm{O}$ : Calculated: $\mathrm{C}, 83.63$; H, 5.81; N, 6.73; Found: C, 83.64; H, 5.80; N, 6.73.

1-Benzyl-2-(3,4-dimethoxyphenyl)-4,5-diphenyl-1H-imidazole (5j). MW: 446.54; $\mathrm{R}_{f}$ : 0.69; FT-IR ( $v_{\max }$; $\left.\mathrm{cm}^{-1} \mathrm{KBr}\right): 3073,2955$ (C- $\left.\mathrm{H}_{\text {stretch }}\right), 1659$ (C=N $\left.\mathrm{N}_{\text {stretch }}\right), 1218\left(\mathrm{C}-\mathrm{N}_{\text {stretch }}\right), 881 \mathrm{~cm}^{-1} ;{ }^{1} \mathrm{H}-\mathrm{NMR}(400 \mathrm{MHz}$, $\mathrm{CDCl}_{3}$ ) $\delta$, ppm: 7.53-7.39 (d, 2H J = 8.7 Hz, Ar-H), 7.79-7.24 (m, 15H, Ar-H), 6.94 (s, 1H, Ar-H), 5.57 $\left(\mathrm{s}, 2 \mathrm{H}, \mathrm{CH}_{2}\right), 3.83\left(\mathrm{~s}, 6 \mathrm{H}, \mathrm{OCH}_{3} \times 2\right) ;{ }^{13} \mathrm{C}-\mathrm{NMR}\left(100 \mathrm{MHz} \mathrm{CDCl}_{3}\right) \delta, \mathrm{ppm}: 153.7,150.3,149.8,141.3$, 138.2, 137.3, 133.1, 129.6, 129.1, 128.8, 128.4, 127.6, 127.3, 125.7, 123.9, 122.3, 112.3, 111.4, 56.2, 47.6; Mass spectrum: $447.56(\mathrm{M}+1)$; Elemental analysis for $\mathrm{C}_{30} \mathrm{H}_{26} \mathrm{~N}_{2} \mathrm{O}_{2}$ : Calculated: $\mathrm{C}$, 80.69; $\mathrm{H}, 5.87$; N, 6.27; Found: C, 80.71; H, 5.88; N, 6.27 .

\subsection{Pharmacology}

\subsubsection{Corneal Anesthesia}

Male New Zealand rabbits were used for the determination of local anesthetic activity of the synthesized compounds. Local surface anesthesia was evaluated by determining the number of stimuli to the cornea every $3 \mathrm{~min}$. [25] This was affected rhythmically with a Frey's horse-hair, in order to produce the blink reflex. If the reflex did not occur after 100 stimulations, anesthesia was considered total. At the beginning of the experiment, special care was taken to ascertain that this reflex was normal in both eyes of the rabbits used. The aqueous solutions $(2 \%)$ of the compounds studied were dropped onto the conjunctival sac so that the space between the eyelids contained a clearly visible film of solution for the set time of $3 \mathrm{~min}$. Lidocaine solution (2\%) was used as standard for comparison.

\subsubsection{Mouse Tail Anesthesia}

Male Swiss mice (weighing 18-20 g) were used. The test was performed according to the method of Bianchi in which the aqueous anesthetic solution $(0.1 \mathrm{~mL})$ is injected $\mathrm{SC}$ about $1 \mathrm{~cm}$ from the root of the tail [26]. Fifteen minutes after injection, the pain reflex of all the injected animals was tested applying a small artery clip to the zone where the compound was injected. The proportion of animals which do not show the usual pain reflex within $30 \mathrm{~s}$ was noted for each dose. Lidocaine solutions were used for comparison. $\mathrm{IC}_{50}$ values were calculated for each compound by probit analysis using a computer program [27].

\subsubsection{Rat Sciatic Nerve Block}

Triplicate sets of three groups of three male Wistar rats (weighing 180-200 g) were used, according to the method as outlined by Al-Saadi and Sneader [28]. Each rat received an injection $(0.2 \mathrm{~mL})$ of the aqueous anesthetic solution $(2 \%)$ into the posterior aspect of the femur head. A positive effect of the drug resulted in a complete loss of motor control of the injected limb. In order to assess the duration of the effect, the animals were observed from the time of onset of the motor paralysis at $5 \mathrm{~min}$ intervals for the first $30 \mathrm{~min}$, and at $15 \mathrm{~min}$ intervals after that up to the first sign of motor activity.

\subsubsection{Acute Toxicity}

The intra paritoneal acute toxicity of the most active compound $\mathbf{5 g}$ was determined in male Swiss mice weighing $18-20 \mathrm{~g} 7$ days after treatment. $\mathrm{LD}_{50}$ values were calculated for each compound by probit analysis using a computer program [27].

\section{Conclusions}

In summary, we have presented a novel, highly rapid and efficient protocol for the synthesis of 1,2,4,5-tetrasubstituted imidazoles as local anesthetic agents via a one-pot condensation reaction. The developed methodology offers an environmentally benign and safe protocol which includes 
a simple reaction setup not requiring specialized equipment, and all the reactions peform well at $100{ }^{\circ} \mathrm{C}$ to afford the desired compounds in excellent yield without use of the solvent. Moreover, the catalyst used in present method can be recovered and recycled making the procedure potentially useful for commercial applications. In bioactivity screening using various methods, some of these compounds were identified as excellent potential local anesthetic agents. Thus, together with efficient methodology of synthesis and pronounced local anesthetic activity, these compounds may serve as a prospective leads for further drug development.

Acknowledgments: Authors are thankful to the authorities for Zhongnan Hospital of Wuhan University, Wuhan University, China for providing necessary facilities for this project. Authors are also thankful to Custom Synthesis Corporation, Anderson, CA, USA for extending technical support for the synthesized compounds reported herein.

Author Contributions: Y.R. and M.L. designed, synthesized and characterized the compounds. Z.-Z.Z. performed local anesthetic and toxicity activity assays.

Conflicts of Interest: The authors declare no conflict of interest.

\section{References}

1. Dua, R.; Shrivastava, S.; Sonwane, S.K.; Srivastava, S.K. Pharmacological significance of synthetic heterocycles scaffold: A Review. Adv. Biol. Res. 2011, 5, 120-144.

2. Zhang, L.; Peng, X.M.; Damu, G.L.; Geng, R.X.; Zhou, C.H. Comprehensive review in current developments of imidazole-based medicinal chemistry. Med. Res. Rev. 2014, 34, 340-437. [CrossRef] [PubMed]

3. Wu, T.; Nagle, A.; Kuhen, K.; Gagaring, K.; Borboa, R.; Francek, C.; Chen, Z.; Plouffe, D.; Goh, A.; Lakshminarayana, S.B.; et al. Imidazolopiperazines: Hit to lead optimization of new antimalarial agents. J. Med. Chem. 2011, 54, 5116-5130. [CrossRef] [PubMed]

4. Saravanan, S.; Selvan, P.S.; Gopal, N.; Gupta, J.K.; De, B. Synthesis and antibacterial activity of some Imidazole-5-(4H)one derivatives. Arch. Pharm. 2005, 338, 488-492. [CrossRef] [PubMed]

5. Schilling, J.C.; Adamus, W.S.; Kuthan, H. Antihistaminic activity and side effect profile of epinastine and terfenadine in healthy volunteers. Int. J. Clin. Pharmacol. Ther. Toxicol. 1990, 28, 493-497. [PubMed]

6. Silva, V.G.; Silva, R.O.; Damasceno, S.R.; Carvalho, N.S.; Prudêncio, R.S.; Aragão, K.S.; Guimarães, M.A.; Campos, S.A.; Véras, L.M.; Godejohann, M.; et al. Anti-inflammatory and antinociceptive activity of epiisopiloturine, an imidazole alkaloid isolated from Pilocarpus microphyllus. J. Nat. Prod. 2013, 76, 1071-1077. [CrossRef] [PubMed]

7. Sondhi, S.M.; Jain, S.; Dinodia, M.; Kumar, A. Synthesis of some thiophene, imidazole and pyridine derivatives exhibiting good anti-inflammatory and analgesic activities. Med. Chem. 2008, 4, 146-154. [CrossRef] [PubMed]

8. Pandey, J.; Tiwari, V.K.; Verma, S.S.; Chaturvedi, V.; Bhatnagar, S.; Sinha, S.; Gaikwad, A.N.; Tripathi, R.P. Synthesis and antitubercular screening of imidazole derivatives. Eur. J. Med. Chem. 2009, 44, 3350-3355. [CrossRef] [PubMed]

9. Aguirre, G.; Boiani, M.; Cerecetto, H.; Gerpe, A.; González, M.; Sainz, Y.F.; Denicola, A.; de Ocáriz, C.O.; Nogal, J.J.; Montero, D.; et al. Novel antiprotozoal products: Imidazole and benzimidazole N-oxide derivatives and related compounds. Arch. Pharm. 2004, 337, 259-270. [CrossRef] [PubMed]

10. Dutta, S. Synthesis and anthelmintic activity of some novel 2-substituted-4,5-diphenyl imidazoles. Acta Pharm. 2010, 60, 229-235. [CrossRef] [PubMed]

11. Tao, R.; Tamas, G.; Xue, L.; Simon, S.L.; Quitevis, E.L. Thermophysical properties of Imidazolium-based ionic liquids: The effect of aliphatic versus aromatic functionality. J. Chem. Eng. Data 2014, 59, 2717-2724. [CrossRef]

12. Balalaei, S.; Arabanian, A. One-pot synthesis of tetrasubstituted imidazoles catalyzed by zeolite HY and silica gel under microwave irradiation. Green Chem. 2000, 2, 274-276. [CrossRef]

13. Karimi, A.R.; Alimohammadi, Z.; Azizian, J.; Mohammadi, A.A.; Mohammadizadeh, M.R. Solvent-free synthesis of tetrasubstituted imidazoles on silica gel/ $\mathrm{NaHSO}_{4}$ support. Catal. Commun. 2006, 7, 728-732. [CrossRef] 
14. Kantevari, S.; Vuppalapati, S.V.N.; Biradar, D.O.; Nagarapu, L. Highly efficient, one-pot, solvent-free synthesis of tetrasubstituted imidazoles using $\mathrm{HClO}_{4}-\mathrm{SiO}_{2}$ as novel heterogeneous catalyst. J. Mol. Catal. A Chem. 2007, 266, 109-113. [CrossRef]

15. Kidwai, M.; Mothsra, P.; Bansal, V.; Somvanshi, R.K.; Ethayathulla, A.S.; Dey, S.; Singh, T.P. One-pot synthesis of highly substituted imidazoles using molecular iodine: A versatile catalyst. J. Mol. Catal. A Chem. 2007, 265, 177-182. [CrossRef]

16. Sadeghi, B.; Mirjalili, B.B.F.; Hashemi, M.M. $\mathrm{BF}_{3} \cdot \mathrm{SiO}_{2}$ : An efficient reagent system for the one-pot synthesis of 1,2,4,5-tetrasubstituted imidazoles. Tetrahedron Lett. 2008, 49, 2575-2577. [CrossRef]

17. Sharma, D.; Hazarika, P.; Konwar, D. An efficient and onepot synthesis of 2,4,5-trisubstituted and 1,2,4,5-tetrasubstituted imidazoles catalyzed by $\mathrm{InCl}_{3} \cdot 3 \mathrm{H}_{2} \mathrm{O}$. Tetrahedron Lett. 2008, 49, 2216-2220. [CrossRef]

18. Nagarapu, L.; Apuri, S.; Kantevari, S. Potassium dodecatugstocobaltate trihydrate $\left(\mathrm{K}_{5} \mathrm{CoW}_{12} \mathrm{O}_{40} \cdot 3 \mathrm{H}_{2} \mathrm{O}\right)$ : A mild and efficient reusable catalyst for the one-pot synthesis of 1,2,4,5-tetrasubstituted imidazoles under conventional heating and microwave irradiation. J. Mol. Catal. A Chem. 2007, 266, 104-108. [CrossRef]

19. Ziarani, M.G.; Dashtianeh, Z.; Nahad, M.S.; Badiei, A. One-pot synthesis of 1,2,4,5-tetra substituted imidazoles using sulfonic acid functionalized silica $\left(\mathrm{SiO}_{2}-\mathrm{Pr}-\mathrm{SO}_{3} \mathrm{H}\right)$. Arabian J. Chem. 2015, 8, 692-697. [CrossRef]

20. Davoodnia, A.; Heravi, M.M.; Safavi-Rad, Z.; Tavakoli-Hoseini, N. Green, one-pot, solvent-free synthesis of 1,2,4,5-tetrasubstituted imidazoles using a Bronsted acidic ionic liquid as novel and reusable catalyst. Synth. Commun. 2010, 40, 2588-2597. [CrossRef]

21. Shoar, R.H.; Rahimzadeh, G.; Derikvand, F.; Farzaneh, M. Four-component, one-pot synthesis of tetra-substituted imidazoles using a catalytic amount of MCM-41 or p-TsOH. Synth. Commun. 2010, 40, 1270-1275. [CrossRef]

22. Hasaninejad, A.; Zare, A.; Shekouhy, M.; Ameri Rad, J. Catalyst-free one-pot four component synthesis of polysubstituted imidazoles in neutral ionic liquid 1-butyl-3-methylimidazolium bromide. J. Comb. Chem. 2010, 12, 844-849. [CrossRef] [PubMed]

23. Shaterian, H.R.; Ranjbar, M.; Azizi, K. Efficient multicomponent synthesis of highly substituted imidazoles utilizing $\mathrm{P}_{2} \mathrm{O}_{5} / \mathrm{SiO}_{2}$ as a reusable catalyst. Chin. J. Chem. 2011, 29, 1635-1645. [CrossRef]

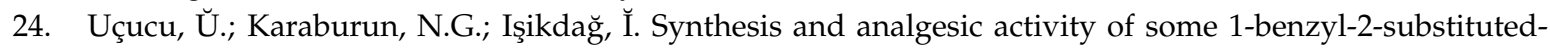
4,5-diphenyl-1H-imidazole derivatives. IL Farmaco 2001, 56, 285-290.

25. Regnier, J. Essai de mesure de l'anesthésie produite sur les terminaisons nerveuses (cornée, muqueuse linguale) par les anesthésiques locaux. Comparaison des pouvoirs anesthésiques. Bull. Sci. Pharm. 1923, 30, 580-586.

26. Bianchi, C. A simple new quantitative method for testing local anaesthetics. Br. J. Pharmacol. 1956, 11, 104-106. [CrossRef]

27. Tallarida, R.J.; Murray, R.B. Manual of Pharmacologic Calculations with Computer Programs, 2nd ed.; Springer: New York, NY, USA, 1981.

28. Al Saadi, D.; Sneader, W.E. Pharmacological evaluation of certain novel prolonged-acting local anaesthetics. In vivo rat sciatic nerve block. Arzneim Forsch. 1991, 41, 195-198.

Sample Availability: Samples of the compounds $\mathbf{5 a - 5 j}$ are available from the authors.

(C) 2015 by the authors; licensee MDPI, Basel, Switzerland. This article is an open access article distributed under the terms and conditions of the Creative Commons by Attribution (CC-BY) license (http://creativecommons.org/licenses/by/4.0/). 